\title{
Differential Many-Body Effects for Initial and Core-Ion States: Impact on XPS Spectra*
}

\author{
Paul S. Bagus, ${ }^{a}$ Carmen Sousa, ${ }^{b}$ and Francesc Illas ${ }^{b}$ \\ ${ }^{a}$ Department of Chemistry, University of North Texas, Denton, TX 76203-5017, USA \\ ${ }^{b}$ Departament de Ciència de Materials i Química Física \& Institut de Química Teòrica i \\ Computacional (IQTCUB), Universitat de Barcelona, C/Martí i Franquès 1, \\ 08028 Barcelona, Spain
}

\begin{abstract}
.
In this paper, the contribution of many body effects to the X-ray photoelectron spectroscopy, XPS, of an NO molecule are studied using wavefunction theory where the specific consequences of different many-body terms are examined and contrasted. It is shown that there is a differential importance of the many-body effects for the different configurations involved in the XPS. These are the ground, initial state configuration and final, $\mathrm{N}(1 \mathrm{~s})$ and $\mathrm{O}(1 \mathrm{~s})$ core-hole ionic configurations. The consequences of the many-body effects are examined for the binding energies, BEs, to the two final state multiplets, triplet and singlet, for each of the core ions and for the relative intensities of the XPS transitions to these multiplets. The many body effects examined are those described as static effects that arise for individual terms that are important. The objective is to understand the chemical and physical origins that determine the importance of the correlation effects for the XPS, rather than to obtain very accurate predictions of the BEs. An important theoretical construct that is tested and justified is the equivalent core approximation where the core ionized atom is replaced by the next higher element in the periodic table. This construct allows us to establish a correlation for the relative importance of the many-body effects in terms of effective charges of the different atoms. This is a correlation that has not been considered before and that we expect may have general relevance. The potential of the effects that we have identified for the XPS of NO to be relevant for the XPS of more complex, condensed phase systems is considered.
\end{abstract}

Keywords: XPS, Static Correlation, Equivalent Core Model

*Dedicated to Prof. Jean Paul Malrieu on occasion of his $80^{\text {th }}$ birthday 


\section{Introduction}

It is common to regard X-Ray Photoelectron Spectroscopy, XPS, from a one-electron point of view where an electron in a core-level is photo-ionized but the other electrons retain the same configuration; see, for example, Refs. [1-4]. Of course, the orbitals of the hole state configuration are different from those of the initial state since they respond to the presence of the core-hole on one of the atoms in the system; this is normally described as a screening or relaxation process [5]. However, for the XPS main peaks, the orbital occupations of the valence levels are viewed as being the same as in the initial, or ground, state before the core ionization and this is the reason to regard XPS as a one-electron process. On the other hand, it is well known that there are many-electron effects that may lead to major changes from a one-electron description, especially for open-shell systems; see, for example, Ref. [5]. One of the important effects leading to additional peaks is the angular momentum coupling of the open core shell that has been photoionized and the electrons in the valence open shell; this angular momentum coupling leads to multiplets with splittings that are often observed; see, for example, Ref. [6] and references therein. Indeed, these multiplets, in particular their energy splittings and relative intensities, may be a way to characterize the oxidation state of a compound as originally pointed out by Gupta and Sen [7,8]. However, even for these multiplets, the occupations of the closed and open shells, except for the ionized core level, do not change between the initial and core-ionized, final configurations.

There are additional classes of many-body effects where the wavefunctions are no longer single configurations but are mixtures of configurations, usually referred to as configuration interaction or CI, with different occupations of orbitals in a space described as an active orbital space [6].This type of many-body effect was used to explain and interpret the $3 \mathrm{~s}$ XPS of MnO [9] where configurations of $\mathrm{Mn}^{2+}$ of the form $3 s^{2} 3 p^{4} 3 d^{6}$ were mixed with the $3 s^{1} 3 p^{6} 3 d^{5}$ XPS allowed configuration. This CI mixing led to a dramatic change in the splitting and relative intensities of the multiplets of the one-electron configuration. Additional configurations with an enlarged active space of orbitals improved the theoretical 3s-hole multiplet splitting compared to experiment and also gave a 3s binding energy, BE, consistent with experiment [10]. These types of many-body, or electron correlation, effects were described as involving near degeneracy between the different configurations formed from different occupations of the active orbitals. They are characterized as being static correlation effects as contrasted with dynamical correlation effects where large orbital and configuration spaces are used [11,12]. An important distinction is 
that static correlation effects involve individual terms that make large contributions to the wavefunction and energy of a system while dynamic correlation effects involve a large number of configurations each with small contributions to the wave function but are needed to obtain very high accuracies. Closely related types of static correlation effects were shown to be important for the $3 \mathrm{~s}$ XPS of the Ar atom and the 2s XPS of the Ne atom [13]. In this paper, our concern is for static many-body effects and, in particular, we shall relate these effects to the chemical interactions.

The many-body effects described for the 3s XPS of Mn in MnO involved purely atomic effects and did not directly involve the chemical bonding. Still another type of static correlation does involve the character of the chemical bond and this was used to correct deficiencies of the Hartree-Fock, HF, wavefunctions for the CO molecule. [14] Here, the extension from the earlier work for $\mathrm{MnO}$ was to consider the static correlation of bonding electrons in the molecule while the XPS was for a core-level electron. In particular, the correlation of the valence, bonding electrons was different for the initial, neutral configuration and for the configurations of the ionized $\mathrm{CO}$ where a core-level electron was removed. The different correlation of the valence levels made it possible to understand the anomalous error of the $\mathrm{HF} C(1 \mathrm{~s}) \mathrm{BE}$ which was larger than the measured BE. It is expected that the HF energy of an ion, with fewer electron pairs, have a smaller error than the HF energy of the neutral, initial state and, thus, the HF BE will be smaller than the observed BE $[14,15]$. When the calculated HF BE is larger than experiment, then the correlation energy must be larger in the core-ion than in the neutral, initial state without a corehole. This anomalous situation can, with suitable analysis, provide insight into the character of the chemical bonding in both the initial and the final, core-ion configurations. Indeed, besides unexpected values of BEs, it will be shown in this paper, that these molecular, chemical bonding, many-body effects may also modify intensities, especially between different final multiplets. Furthermore, information can flow in two directions; as well as using unexpected XPS features to gain insight into the chemistry of a material, understanding the chemistry may make it possible to predict unexpected features in the XPS.

In the present paper, the previous work on molecular, valence level, many-body effects for the XPS of the closed shell CO molecule is extended to analyze and interpret the XPS of the open-shell NO molecule. There are several aspects to the present extension. First, we examine whether and how the many-body effects are different for the different final state multiplets of NO for the core-level ions. Since the ground state of NO is ${ }^{2} \Pi$, the XPS allowed multiplets for either 
$\mathrm{N}(1 \mathrm{~s})$ or $\mathrm{O}(1 \mathrm{~s})$ ions are ${ }^{3} \Pi$ or ${ }^{1} \Pi$. Thus, as well as determining the consequences of the manybody effects for the absolute core-level BEs; their effect on the relative energies of the ${ }^{3} \Pi$ and ${ }^{1} \Pi$ multiplets is also considered. In addition to the relative BEs of these multiplets, the relative XPS intensities of the multiplets are also considered. In the simplest approximation, the intensity ratio of different multiplets is given by the multiplicity $[5,16]$, which for the NO multiplets would be $\mathrm{I}\left({ }^{3} \Pi\right) / \mathrm{I}\left({ }^{1} \Pi\right)=3$. However, the intensity ratio for the $N(1 \mathrm{~s})$ multiplets is $15 \%$ larger than $3[16]$ and only a small fraction of this difference could be explained with HF wavefunctions. The introduction of many-body effects resolves a long-standing absence of an explanation for the observed departure of the XPS intensity ratio of the N(1s) multiplets. Second, the active orbital space for the static many-body effects used in the present analysis is extended over that used previously and now includes $\sigma$ as well as $\pi$ orbitals. This extension allows the testing of the importance of different static correlation effects. Finally, the equivalent core, EQC, approximation $[17,18]$, has been used to provide a chemical understanding of the differences in the many-body effects between the neutral initial state of $\mathrm{NO}$ and the $\mathrm{N}(1 \mathrm{~s})$ and $\mathrm{O}(1 \mathrm{~s})$ core ionic states. Within the EQC model, the core ionized atom is replaced by the next atom in the periodic table. Thus, when an $\mathrm{N}(1 \mathrm{~s})$ electron is removed, the ionized molecule is consider as equivalent to $\mathrm{O}_{2}{ }^{+}$where $\mathrm{N}$ has been replaced by $\mathrm{O}$ and for the $\mathrm{O}(1 \mathrm{~s})$ ionization, the equivalent molecule is $\mathrm{NF}^{+}$; for a discussion of the EQC validity, see Ref. [6]. One of the important areas where XPS is used to provide information about and insights into materials properties is for condensed matter; see, for example, Refs. $[5,6]$ and references therein. Although our analysis is for an isolated molecule, we believe that the many body effects that are shown to be important for NO are also relevant for condensed systems. However, since it is more straightforward to explore and test these manybody effects for a simpler, isolated molecule than for a solid system, the present work, albeit valuable in its own right, lays the foundation and provides guidance for future studies of condensed systems and the potential importance of the many-body features that have been identified for the NO molecule to the analysis of the XPS of condensed systems, especially ionic compounds, is considered.

\section{Theoretical Formulations and Methodology}

Since $\mathrm{N}$ and $\mathrm{O}$ are light atoms, the major theoretical methods used to determine wavefunctions and their energies and properties are non-relativistic; the estimate of relativistic corrections for the $\mathrm{N}(1 \mathrm{~s})$ and $\mathrm{O}(1 \mathrm{~s})$ BEs is described later in this section. Different methods are 
available to accurately describe BEs as recently reviewed [19]. These include quantum chemical wave function based methods [20], those relying on many body Green's function approaches such as GW, recently applied to the prediction of core level BEs [21], and propagator methods broadly used to study ionization processes [22,23]. In the present work we chose HF and the Complete Active Space, CAS, [24] Self Consistent Field wavefunction based methods which allow for a more direct interpretation in terms of chemical concepts.

For the ground and ionic states both HF and CAS wavefunctions have been computed. In the HF procedure, the orbitals are variationally optimized to give the most accurate single configuration wavefunction. In the CAS procedure, both the orbitals and the configuration mixing coefficients are variationally optimized to give the most accurate wavefunction for the configuration and orbital spaces used were chosen to include the most-important many-body terms; i.e., the static correlation effects $[11,12]$. The fact that the BEs discussed in the next section are very close to the experimental values supports the claim that the contribution of the missing dynamic electron correlation is small and, more important, does not introduce significant new physics. An important property that will be stressed in the analysis of the many-body effects on the XPS is the correlation energy, $\mathrm{E}_{\text {corr }}$, recovered from the CAS wavefunctions over the HF wavefunctions, i.e. $\mathrm{E}_{\text {corr }}=\mathrm{E}(\mathrm{CAS})-\mathrm{E}(\mathrm{HF})$, where $\mathrm{E}_{\text {corr }}$ is computed for the different $\mathrm{NO}$ multiplets and for the different CAS wavefunctions. Hereby, CAS $(4,5)$ and $\operatorname{CAS}(6,7)$ have been used as described in detail in the Electronic Supplementary Material (ESM). In addition, HF and CAS wavefunctions are also calculated for the EQC molecules [17,18], $\mathrm{O}_{2}{ }^{+}$and $\mathrm{NF}^{+}$.

The concern here is for the XPS properties of the main ${ }^{3} \Pi$ and ${ }^{1} \Pi N(1 s)$ and $O(1 s)$; specifically, their binding energies, BEs, and their relative intensities, $\mathrm{I}_{\mathrm{rel}}$. The BEs are computed by taking the differences of the variational HF and CAS energies for the ground state, GS, and the core ionic states. This is referred to as $\mathrm{BE}(\Delta \mathrm{SCF})$, see, for example, Ref. [5], where

$$
\mathrm{BE}(\Delta \mathrm{SCF}, \mathrm{i})=\mathrm{E}(\text { core ion on atom } \mathrm{i})-\mathrm{E}(\mathrm{GS}) \text {. }
$$

The $\mathrm{BE}(\triangle \mathrm{SCF})$ include both initial state effects arising from the different chemical environments of the atom before ionization as well as final state effects arising from the response, primarily of the valence electrons, to the presence of the core hole [5], We do not explicitly distinguish the initial and final state effects. This is because our objective is to examine the difference in many body effects between the ionic configurations and the initial state which is inherently a final state effect. For the relative intensities of the ${ }^{3} \Pi$ and ${ }^{1} \Pi$ XPS peaks, the sudden approximation, SA, $[25,26]$ is used. The essential foundation of the SA $\mathrm{I}_{\mathrm{rel}}$ is that one considers the overlap of the 
frozen orbital wavefunction, where a core electron is removed but the other orbitals are frozen as in the GS, with the fully relaxed variational hole state wavefunctions. The application of the SA to XPS intensities for HF wavefunctions for NO is reviewed in Ref. [16] and the extension to CAS wavefunctions is straightforward. The use of the SA for the XPS of open-shell systems is reviewed in Ref. [5].

For the BEs, relativistic effects are taken into account. While the relativistic contributions for the BEs of light atoms, like $\mathrm{N}$ and $\mathrm{O}$, are not especially large, $\sim 0.5 \mathrm{eV}$ [27], including them allows a better comparison to be made between the errors of the theoretical $\mathrm{N}(1 \mathrm{~s})$ and $\mathrm{O}(1 \mathrm{~s}) \mathrm{BEs}$. While, in principle, it is possible to carry out fully relativistic calculations with program systems like MOLFDIR [28] or DIRAC [29], this could involve considering very small spin-orbit splittings of the initial ${ }^{2} \Pi$ and the final, core ion, ${ }^{3} \Pi$ states [30]. From 4 component Dirac Hartree-Fock, DHF, calculation on NO, these splittings have been determined to be less than 0.02 $\mathrm{eV}$. Thus, it has been chosen to use corrections to non-relativistic HF and CAS BEs based on relativistic Dirac Hartree-Fock, DHF, calculations on the GS and core-level ions of NO. This assumes that the relativistic correction to the CAS BEs is the same as for the HF BEs which should be reasonable since the scalar relativistic effects which lead to changes in the nonrelativistic BEs should be well represented with DHF wavefunctions. In Table 1, the relativistic $\mathrm{BE}$ contributions, $\triangle \mathrm{BE}_{\mathrm{rel}}$, to the $\mathrm{NO} \mathrm{BEs}$ are compared to atomic relativistic contributions to the BEs of isolated $\mathrm{N}$ and $\mathrm{O}$. The $\triangle \mathrm{BE}_{\text {rel }}$ are determined from the difference of relativistic DHF BEs and non-relativistic HF BEs; for both the atoms and NO, the BEs are computed for the average of configurations of the initial and final states and multiplet splittings are neglected [4]. The atomic $\Delta \mathrm{BE}_{\text {rel }}$ are similar to those for $\mathrm{NO}$, which is not surprising. For the BEs reported in Sec. 4, the NO $\Delta \mathrm{BE}_{\mathrm{rel}}$ from Table 1 will be added to the calculated non-relativistic HF and CAS BEs. Relativistic contributions to the SA intensities of the $\mathrm{N}(1 \mathrm{~s})$ and $\mathrm{O}(1 \mathrm{~s})$ hole multiplets will be considered in Sec 4.

A final consideration in this section is how the accuracy of the calculated BEs may be affected because the present calculations are based on the use of Gaussian type basis functions, abbreviated as GTOs, to describe the molecular orbitals of NO [20]. Since GTOs do not have the proper exponential behavior at the nuclei about which they are centered [20], they may have limitations in describing excitations and ionizations of core-orbitals. The BEs of NO represent a case where it is possible to make a rigorous assessment of the accuracy of GTOs since there is an early study of the HF BEs of NO [31] which was based on HF wavefunctions using an extended 
basis set of Slater type basis functions, abbreviated as STOs, which have the flexibility to represent the proper exponential behavior at the nucleus [32]. It was estimated that with the STO basis set used, the total energies of the GS and ionic states were within 0.005 hartree, $0.1 \mathrm{eV}$, of the exact, basis set limit, HF energy [31] and the energy differences can be expected to be even more accurate. While the wavefunction accuracy for the GTO basis sets used is a computational detail, the accuracy for core-level processes that can be obtained with GTO basis sets is of general importance and interest since it concerns the reliability of many theoretical studies [5]. Thus a comparison of the non-relativistic NO BEs obtained with the present GTO basis set and the earlier, accurate STO basis set is given in Table S1 in the ESM. The BEs computed with the GTO basis are all within $0.1 \mathrm{eV}$ of the essentially exact HF BEs computed with the STO basis. In fact, the GTO BEs are too large with respect to the exact HF limit. This means that the GTO error of the ionic state is $0.1 \mathrm{eV}$ larger than the GTO error of the ground state. In other words, the GTO exponents that were optimized for the isolated atoms, see Refs. [33,34], are not as accurate for the core-hole states even when large sets of GTOs are used. However, the most important conclusion from the data in Table S1 is that, with a relatively small uncertainty, large GTO basis sets give reliable core-level BEs.

\section{Computational Details}

The HF and CAS wavefunctions are for the ground state of NO with an open shell configuration of $2 \pi^{1}\left({ }^{2} \Pi\right)$ and for the $\mathrm{O}(1 \mathrm{~s})$ ions, $1 \sigma^{1} \ldots 2 \pi^{1}$, and the $\mathrm{N}(1 \mathrm{~s})$ ions, $2 \sigma^{1} \ldots 2 \pi^{1}$, coupled to ${ }^{3} \Pi$ and ${ }^{1} \Pi$ multiplets. In order to prevent variational collapse to lower lying ionic configurations in the HF calculations of the core ions, the occupied open and closed shell orbitals were selected based on their maximum overlap with trial orbitals with proper character. This approach has been used successfully to determine wavefunctions for core-level ionic and excited states; see, for example, Ref. [5]. For the CAS wavefunctions of the ions a different, freeze-thaw, approach was used to prevent variational collapse to lower lying ionic states. The freeze-thaw method involves pairs of steps; see Ref. [14] for a full description. In the first step, the singly occupied 1s orbital is frozen and the other orbitals are varied in the full basis set space excluding the 1s orbital. In the second step, the other occupied orbitals, as determined in the first step, are frozen and the singly occupied 1s orbital is varied in the full space excluding the other occupied orbitals. The pair of steps is repeated until the CAS wavefunction is converged. Relativistic contributions are added to the HF and CAS non-relativistic BEs. These are determined from 4- 
component relativistic DHF calculations for the GS and ionic states as described in the preceding section. The DHF calculations used a Dirac-Coulomb Hamiltonian without Breit and higherorder corrections with orbitals optimized for the average of configurations. For all calculations, the N-O distance was fixed at the experimental $r_{\mathrm{e}}=2.1747$ bohr [35]. The XPS SA intensities, $\mathrm{I}_{\mathrm{rel}}$, of the ionic multiplets are complicated because the orbitals of the GS are not the same as those for the ${ }^{3} \Pi$ and ${ }^{1} \Pi$ ionic states since the orbitals for these ionic states include the relaxation in response to the core-hole. The necessary evaluation of determinants and minors [36] is obtained using a cofactor method [37].

Large, uncontracted GTO basis sets for $\mathrm{N}$ and $\mathrm{O}$ were used to obtain $\mathrm{HF}$ and CAS wavefunctions and energies that are essentially at the basis set limit and would not change significantly for further increases in the basis set size. They are uncontracted to allow the flexibility to represent the screened core-hole configuration orbitals with the same basis sets as for the GS [4]. The $\mathrm{N}$ basis set contained $14 \mathrm{~s}$ and $9 \mathrm{p}$ basis functions with exponents determined by Partridge [34] and augmented by a d function with exponent of 1.0. The O basis set is based on the $10 \mathrm{~s}$ and $6 \mathrm{p}$ basis set with exponents optimized by Duijneveldt [33] with a $\mathrm{d}$ function of exponent 1.2 added. The quality of this basis set to provide accurate BEs has been discussed in the previous section. The HF wavefunctions and the SA $I_{\text {rel }}$ were calculated using the CLIPS set of programs [38], the CAS wavefunctions were calculated with the programs developed by Roos and Siegbahn [24]. The relativistic DHF wavefunctions were computed with the DIRAC program system [29].

\section{Results and Discussion}

\section{A. $N(1 s)$ and $O(1 s) B E s$}

The theoretical N(1s) and O(1s) BEs for NO are given in Table 2; the BE's in the table for the $\mathrm{HF}, \mathrm{CAS}(4,5)$, and $\mathrm{CAS}(6,7)$ wavefunctions include the relativistic contributions taken from Table 1 and, thus, are appropriate to compare with the experimental XPS results also given in the

table. The N(1s) BEs are discussed first. The HF BEs for both the ${ }^{3} \Pi$ and ${ }^{1} \Pi$ ionic multiplets are larger than experiment, the opposite of the expected error of HF BEs. The HF BEs should be smaller than experiment since, as already discussed, the correlation energy, or the error of HF energies, should be larger for the $\mathrm{N}$ electron initial state compared to the $\mathrm{N}-1$ electron ions [15]. Indeed this is the sign of the error that was found for the N(1s) BEs in a large number of closed shell molecules [39]. BEs larger than experiment mean that the correlation energy is larger for the 
ion than for the initial GS of NO. With the CAS(4,5) wavefunctions static, near degeneracy, many-body (or correlation) effects are included for the $\pi$ electrons within the space of the $1 \pi$ and $2 \pi$ orbitals. These static many body effects are more important for the $\mathrm{N}(1 \mathrm{~s})$ ion than for the initial GS and lower the HF BEs by $\sim 1.4 \mathrm{eV}$. When additional static many-body effects involving the $\sigma$ space as well as the $\pi$ space are included with the CAS $(6,7)$ wavefunctions, the BEs are reduced by another $0.5 \mathrm{eV}$ indicating a further differential many body effect greater for the $\mathrm{N}(1 \mathrm{~s})$ ion than for the GS. The CAS(6,7) BEs are now smaller than experiment by $\sim 0.5 \mathrm{eV}$. It is not surprising that the $\mathrm{CAS}(6,7) \mathrm{BEs}$ are smaller than experiment since we have not included corecore and core-valence many-body effects in our many-body treatment and these effects should be larger for the GS where the core has two N(1s) electrons while the N(1s) ions have only one. The multiplet splitting of ${ }^{3} \Pi$ and ${ }^{1} \Pi$ BEs, $\Delta\left({ }^{1} \Pi-{ }^{3} \Pi\right)$, are reasonably close to experiment for all the wavefunctions indicating that the many-body effects are similar, although clearly not identical, for both ionic multiplets. Indeed, the changes between the HF and CAS multiplet splittings are smaller for the $\mathrm{O}(1 \mathrm{~s})$ ions than for the $\mathrm{N}(1 \mathrm{~s})$ ions. The relevance of this for the XPS relative intensities of the multiplets will be described in subsection $\mathrm{C}$.

The error of the HF BEs and the differential many-body effects are different for the $\mathrm{O}(1 \mathrm{~s})$ core ions. The HF O(1s) BEs are smaller than experiment by $\sim 1 \mathrm{eV}$, as expected indicating that the many body effects are larger for the GS than for the $\mathrm{O}(1 \mathrm{~s})$ ions, the opposite of the case for the $\mathrm{N}(1 \mathrm{~s})$ ions. Furthermore, the $\pi$ many-body effects with $\mathrm{CAS}(4,5)$ wavefunctions raise the BE rather than lowering it as was the case for the N(1s) BEs. In other words, these many body contributions to the energy of the GS are larger than they are for the $\mathrm{O}(1 \mathrm{~s})$ ions. The additional many-body effects, in the $\sigma$ space, introduced with the CAS $(6,7)$ wavefunctions lead to a smaller change in the BEs but in the opposite direction, making the BEs $\sim 0.3 \mathrm{eV}$ smaller. The CAS(6,7) BEs are somewhat less than experiment, $<0.5 \mathrm{eV}$, indicating that the many-body effects not included in this $\pi$ and $\sigma$ treatment of near-degeneracy many-body effects will not have a large differential effect on the energies of the GS and the $\mathrm{O}(1 \mathrm{~s})$ ions. The theoretical multiplet splittings, $\Delta\left({ }^{1} \Pi^{-}{ }^{3} \Pi\right)$, for the $\mathrm{O}(1 \mathrm{~s})$ ions are essentially the same for the $\mathrm{HF}$ and CAS wavefunctions and are quite close to experiment indicating that the differential many-body effects are quite small between the singlet and triplet multiplets.

It is satisfying that the CAS treatments give good agreements with XPS measurements for both the $\mathrm{N}(1 \mathrm{~s})$ and $\mathrm{O}(1 \mathrm{~s})$ ions; in particular, the error of the calculated BEs is that they are 
smaller than the XPS observed values [31]. This sign of error is expected because the core-core and core-valence many body effects are not considered. The BEs in Table 2 should be reduced by $\sim 0.1 \mathrm{eV}$ because our basis set is not fully at the limit that would be reached by a complete basis set; see Table S1 and related discussion in Section 2. This additional change in the BEs is sufficiently small that it can be neglected for the purpose of our theoretical analysis. On the other hand, it is important to obtain an understanding of the origin of the reasonably large differential static correlation effects. This understanding will allow one to predict when such differential effects might be important for the XPS BEs. They may also provide guides to the many-body effects that need to be taken into account for the description of the XPS, not only of NO and other small molecules, but for other systems including those in the condensed phase. This analysis will be made in the following subsection where the properties of the HF and CAS wavefunctions are examined.

\section{B. Properties of the Wavefunctions}

The energy gain of the CAS wavefunctions, $\Delta \mathrm{E}_{\text {corr, }}$, and orbital properties of selected orbitals are given in Table S2 for the GS of NO. The properties are the orbital occupations and the $<\mathrm{z}>$ where $\mathrm{z}$ is the $\mathrm{N}-\mathrm{O}$ axis. The $<\mathrm{z}>$ is taken with respect to the center of the molecule at 1.087 bohrs or $0.575 \AA$ to the right of the $\mathrm{N}$ atom; this choice of origin directly shows the extent of polarization of the orbital toward either $\mathrm{N}$, with $<\mathrm{z}><0$, or toward $\mathrm{O}$ with $<\mathrm{z}>>0$.

The energy gain for the CAS(4,5) is $1.61 \mathrm{eV}$, see Table S2, showing the importance of the $\pi$ static many-body effect of mixing different occupations of the $1 \pi$ and $2 \pi$ orbitals. The additional energy gain going from $\operatorname{CAS}(4,5)$ to $\operatorname{CAS}(6,7)$ is almost as large, over $80 \%$, as the gain from $\mathrm{HF}$ to $\mathrm{CAS}(4,5)$ showing that the static many-body effects in the $\sigma$ space are also important. Although exchange of electrons between the $\sigma$ and $\pi$ spaces is allowed in the $\operatorname{CAS}(6,7)$ wavefunction, the occupations of electrons in the $\sigma$ and $\pi$ active spaces remain very close to 2 and 5, respectively, see Table S2. This shows that the $\sigma$ active space treats many body effects for the $5 \sigma$ orbital and does not contribute to many-body effects in the $\pi$ space. For the HF wavefunction, the $1 \pi$ and $2 \pi$ are bonding polarized toward $O$ and anti-bonding polarized toward $\mathrm{N}$, respectively. This polarization is reduced for the CAS wavefunctions where there is also a promotion of 0.1 e from $1 \pi$ to $2 \pi$. The $5 \sigma$ orbital is bonding and polarized toward $\mathrm{N}$ for the HF and $\operatorname{CAS}(4,5)$ wavefunctions. For the $\operatorname{CAS}(6,7)$ wavefunction, there is a small promotion of 
$0.02 \mathrm{e}$ from the bonding $5 \sigma$ to anti-bonding $6 \sigma$. The character of the orbitals is shown by contour plots in Figs. S1 and S2 of the active CAS(6,7) orbitals, see additional detail on the ESM.

Very different properties of the HF and CAS wavefunctions for the N(1s) ion multiplets are shown in Table S3. The properties of the ${ }^{3} \Pi$ and ${ }^{1} \Pi$ multiplets are similar, albeit not identical, and different from those for the NO GS. The $<\mathrm{z}>$ show a major valence relaxation in the active orbital space in response to the $\mathrm{N}(1 \mathrm{~s})$ ionization. In particular, the $\pi$ orbitals are now much more evenly distributed between $\mathrm{N}$ and $\mathrm{O}$ with values of $\langle\mathrm{z}>$, with respect to the center of $\mathrm{NO}$, that are nearly zero. The HF and CAS $(4,5) 5 \sigma<\mathrm{z}>$ while not quite zero are about half what they were for the GS, and, as for the GS, the $\operatorname{CAS}(6,7) 5 \sigma$ and $6 \sigma<\mathrm{z}>$ are nearly zero, indicating that these orbitals are not strongly polarized toward either $\mathrm{N}$ or $\mathrm{O}$; see Tables $\mathrm{S} 2$ and $\mathrm{S} 3$. This is consistent with the fact that, by symmetry, the orbitals of the EQC molecule $\mathrm{O}_{2}^{+}$, also shown in Table S3, where the core ionized $\mathrm{N}$ is replaced by an $\mathrm{O}$ atom $[17,18]$, have $<\mathrm{z}>=0$. In other words, the valence electrons for the $\mathrm{N}(1 \mathrm{~s})$ ions of NO have a distribution rather similar to that for the equivalent core molecule $\mathrm{O}_{2}{ }^{+}$. Furthermore, the many-body effects for the $\mathrm{N}(1 \mathrm{~s})$ ions and for $\mathrm{O}_{2}{ }^{+}$ are also similar with the occupation numbers of the active space $\pi$ and $\sigma$ being the same within better than $5 \%$ and with the energy lowerings, $\Delta \mathrm{E}_{\text {corr }}$ for the static many-body effects being the same within 3\%. However, the energy changes for the N(1s) ions are larger than for the GS; the many-body effects in the $\pi$ space, accounted for with $\operatorname{CAS}(4,5)$, are larger by almost a factor of 2 and the additional $\sigma$ many-body effects, included with $\mathrm{CAS}(6,7)$, are larger by more than $50 \%$. The larger many-body lowering of the energy of the ionic states is what leads to the major decreases in the $\mathrm{N}(1 \mathrm{~s})$ BEs reported in Table 2. The similarity of the many body effects for the $\mathrm{N}(1 \mathrm{~s})$ ions and the EQC $\mathrm{O}_{2}{ }^{+}$molecule indicate that a knowledge of the valence shell many-body effects of the GS of a molecule and of the EQC molecule will allow us to make an informed estimate of the errors in calculated BEs. There is also support for the understanding that excitations among bonding and anti-bonding orbitals are the key many-body effects to be taken into account for the analysis of XPS spectra.

A parallel analysis of many-body effect for the $\mathrm{O}(1 \mathrm{~s})$ ions, given in Table S4, further supports the general importance of differential static many-body effects in the valence shell. It also shows that these differential many-body effects may lead to increases in the BEs over HF values as well as decreases as was the case for the $N(1 \mathrm{~s})$ ions. The polarization of the $1 \pi$ and $2 \pi$ orbitals in response to the $\mathrm{O}(1 \mathrm{~s})$ core-hole is rather large for the HF and CAS wavefunctions for both the ${ }^{3} \Pi$ and ${ }^{1} \Pi$ multiplets. This polarization is greatest for the HF orbitals where the centers 
of charge of the $1 \pi$ and $2 \pi$ orbitals are $75 \%$ toward the $O$ and $N$ centers, respectively; while, the polarization of these orbitals is reduced for the CAS wavefunctions, they are still much more polarized than for the ground state; see Table S2. For the HF and CAS $(4,5)$ wavefunctions, where many-body effects for the $\sigma$ orbitals are not considered, the center of charge of the $5 \sigma$ is essentially at the $\mathrm{N}$ atom. For the $\mathrm{CAS}(6,7)$ wavefunction, the $5 \sigma$ and $6 \sigma$ orbitals are much less polarized and their center of charge is near the mid-point between $\mathrm{N}$ and $\mathrm{O}$. This rearrangement of charge can be viewed as a response where the $41 \pi$ electrons are attracted toward the $\mathrm{O}$ center with the core-hole and the $2 \pi$ and $5 \sigma$ electrons move toward $N$ to reduce the imbalance of charge. The reduced polarization when $\sigma$ many-body effects are included with the CAS(6,7) wavefunctions may arise because this leads to larger off-diagonal Hamiltonian matrix elements between the configurations introduced with these static $\sigma$ many body effects. One could argue that the off-diagonal matrix elements are larger when the $5 \sigma$ and $6 \sigma$ are bonding and anti-bonding about the center of the molecule rather than having lone pair character about the $\mathrm{N}$ and $\mathrm{O}$ centers, respectively. For the $\operatorname{CAS}(6,7)$ wavefunctions, the inactive $3 \sigma$ and $4 \sigma$ orbitals, not shown, rearrange to mostly offset the motion of $\sigma$ charge in the active $5 \sigma$ and $6 \sigma$ space. The rearrangement of the valence orbitals are similar between the $\mathrm{O}(1 \mathrm{~s})$ ions and the EQC molecule $\mathrm{NF}^{+}$again showing that the EQC model describes the driving forces for the charge rearrangement in response to core hole even if it does not provide a quantitative description of the charge rearrangement or response to the core-holes [5].

The $\pi$ static many-body effects in the $\operatorname{CAS}(4,5)$ wavefunction are smaller for the $\mathrm{O}(1 \mathrm{~s})$ ions than for either the ground state or the $\mathrm{N}(1 \mathrm{~s})$ ions; they lead to an energy improvement which is about half of that for the GS, see Table S2. This explains why the $\mathrm{O}(1 \mathrm{~s})$ BEs with the CAS $(4,5)$ wavefunctions are larger, by $\sim 0.75 \mathrm{eV}$ than the BEs with the HF wavefunctions, see Table 2 . This reduced importance of the $\pi$ many-body effects can also be seen from the occupation numbers for the $1 \pi$ and $2 \pi$ orbitals for the CAS wavefunctions for the $\mathrm{O}(1 \mathrm{~s})$ ions, Table S4. The $1 \pi$ occupation is closer to 4 than for either the GS, Table 3, or the N(1s) ions, Table S3; further, the $\mathrm{N}(1 \mathrm{~s})$ ions have the smallest $1 \pi$ occupation numbers and the largest energy lowering from $\pi$ many-body effects. In contrast, the $\sigma$ many-body effects introduced with the CAS(6,7) wavefunctions for the $\mathrm{O}(1 \mathrm{~s})$ ions are greater than for the GS and smaller than for the $\mathrm{N}(1 \mathrm{~s})$ ions. This means that the $\mathrm{O}(1 \mathrm{~s}) \mathrm{BEs}$ obtained with the CAS $(6,7)$ wavefunctions are smaller than those 
obtained with the CAS $(4,5)$ wavefunctions, reducing the net increase over the HF BEs to $\sim 0.5$ $\mathrm{eV}$.

Using the equivalent core model, there is a correlation that can be drawn for the manybody effects in NO. When the charge separation between the effective atomic charges of the atoms is greater, then the HF one-electron model is better and many-body effects lead to smaller lowerings of the total energy of the system. Thus, the many body effects are greater for the N(1s) ions of $\mathrm{NO}$ where the equivalent core model is $\mathrm{O}_{2}{ }^{+}$and the charge separation is zero than for the NO GS where the charge separation is 1; this will lead to HF BEs that are larger than CAS BEs where the many-body effects are taken into account. On the other hand, for the $\mathrm{O}(1 \mathrm{~s})$ ions of NO where the equivalent core model is $\mathrm{NF}^{+}$and the charge separation is 2 , the many body effects will be smaller than for the NO GS and the HF BEs will be smaller than those where many body effects are included. This same correlation holds for the core-level BEs of CO although this correlation was not recognized in our previous work [14]. There, the C(1s) CAS BE which includes many body effects is smaller than the HF BE and the charge separation in the EQC molecule $\mathrm{NO}^{+}$is 1 or less than the charge separation of 2 for the GS of CO. For the $\mathrm{O}(1 \mathrm{~s})$ ion of $\mathrm{CO}$, the EQC molecule is $\mathrm{CF}^{+}$where the charge separation is 3 or larger than the value of 2 for the $\mathrm{CO}$ GS, the correlation would be that the CAS O(1s) BE is larger than the HF O(1s) BE and this correlation is seen to hold.

\section{XPS Intensities of the ${ }^{3} \Pi$ and ${ }^{1} \Pi$ Multiplets For N(1s) Ions}

Many-body effects on the wavefunctions may affect other properties of XPS spectra besides energies of the core-level ions. In particular, the intensity of the different $\mathrm{N}(1 \mathrm{~s})$ and $\mathrm{O}(1 \mathrm{~s})$ multiplets may not follow the simple rule that the relative intensities of different angular momentum couplings of core and valence open shells follow their multiplicities [16,40]. Indeed, the relative XPS intensity of the $\mathrm{N}(1 \mathrm{~s}){ }^{3} \Pi$ to ${ }^{1} \Pi$ was found to be $3.43: 1$ or $14 \%$ larger than the expected 3:1 statistical ratio while HF wavefunctions could only account for a small fraction of this departure from the statistical data. [16] In Table 3, the Sudden Approximation, SA, relative intensities, $\mathrm{I}_{\mathrm{rel}}$, see Refs. [26] and [5], are given for the $\mathrm{N}(1 \mathrm{~s})$ multiplets for the HF and CAS wavefunctions and compared to experiment. The SA $\mathrm{I}_{\text {rel }}$ are normalized to the ideal values of 3.0

and 1.0 for ${ }^{3} \Pi$ and ${ }^{1} \Pi$ respectively so that the differences from these ideal values give the intensity losses from the main peaks to many-body shake excited states [26] and the losses are shown in Table 3; the ratios of the ${ }^{3} \Pi$ to ${ }^{1} \Pi I_{\text {rel }}$ are also shown in the table. The HF losses to shake satellites are $\sim 25 \%$ from both the ${ }^{3} \Pi$ and ${ }^{1} \Pi$ XPS peaks and slightly larger for the ${ }^{1} \Pi$ peak 
leading to an intensity ratio of 3.09; larger than the ideal ratio of 3.0 but smaller than the observed ratio of 3.43:1. For the CAS $(4,5)$ wavefunctions where only $\pi$ static many-body effects are considered, the ${ }^{3} \Pi$ loss increases slightly to $27 \%$ but the ${ }^{1} \Pi$ loss increases from $26 \%$ to $36 \%$ and the intensity ratio is now 3.41 , close to the measured value. The ${ }^{3} \Pi$ intensity loss for the CAS $(6,7)$ wavefunction increases slightly but the ${ }^{1} \Pi$ loss is almost constant leading to a decrease of the intensity ratio which is still much larger than the HF value and much closer to experiment than HF. The reason for the departures in the intensity ratio from the ideal value is two-fold, partly from the different HF or CAS orbitals for the different multiplets and partly from the different many-body contributions to the CAS wavefunctions to the multiplets. For the HF wavefunctions, the departure from the ideal intensity ratio arises only because the ${ }^{3} \Pi$ and ${ }^{1} \Pi$ orbitals are different. The differences do not have to be especially large since the overlap integrals between the ground state and the ionic state orbitals enter to rather high powers for the SA $I_{\text {rel }}$ and small differences can lead to modest changes in the intensity losses to the shake satellites $[16,26]$. The changes in the ratio from the HF to the CAS $I_{\text {rel }}$ come about 1/3 from the differences in the variational orbitals for the two multiplets and about $2 / 3$ difference in the CAS wavefunctions for the different multiplets; see Table S3 for differences in the properties of the ${ }^{3} \Pi$ and ${ }^{1} \Pi$ wavefunctions. The relativistic contribution to the intensity ratio is expected to arise from the spin-orbit splitting of the initial state ${ }^{2} \Pi$ multiplet of NO into $\Omega=1 / 2$ ad $3 / 2$ levels [30]. Although this splitting is only $0.02 \mathrm{eV}$; the Boltzmann populations of the two levels is, at room temperature, different by over a factor of 2 . The importance of this splitting on the intensity ratio of the multiplets was investigated with Dirac Hartree-Fock complete open shell configuration interaction, COSCI, wavefunctions for the initial and $\mathrm{N}(1 \mathrm{~s})$ ionic configurations which take the spin-orbit splittings into account. The effect was to increase the ratio by $1 \%$ from 3 to 3.03 . A negligible change.

The intensity ratio of the multiplets for the $\mathrm{O}(1 \mathrm{~s})$ ions, is difficult to measure experimentally given the small multiplet splittings, see Table 2, but can be determined from the $\mathrm{HF}$ and CAS wavefunctions. Results for the SA $\mathrm{I}_{\text {rel }}$ and losses to shake excitations for the $\mathrm{O}(1 \mathrm{~s})$ ions, parallel to those for the N(1s) ions in Table 3, are presented in Table 4. The intensity losses of the HF wavefunctions are similar as for the N(1s) case although they are slightly larger for the $\mathrm{O}(1 \mathrm{~s})$ ionization reflecting the increased screening to the hole on $\mathrm{O}$ with a greater nuclear charge. The intensity ratio of ${ }^{3} \Pi$ to ${ }^{1} \Pi$ is virtually the same as for the $N(1 \mathrm{~s})$ ions indicating a similar 
differential screening and intensity loss to shake excitations for the different multiplets in both the $\mathrm{N}(1 \mathrm{~s})$ and $\mathrm{O}(1 \mathrm{~s})$ ions. The difference between the $\mathrm{N}(1 \mathrm{~s})$ and $\mathrm{O}(1 \mathrm{~s})$ ions arises when manybody effects are considered. There were major changes in the intensity ratio going from HF to CAS for the $N(1 s){ }^{3} \Pi$ and ${ }^{1} \Pi$ multiplets but not for the $O(1 s)$ ions. The reason for the smaller many-body effect on the intensity ratio for the $\mathrm{O}(1 \mathrm{~s})$ ions is twofold. First, the magnitude of the many body effects for the $\mathrm{O}(1 \mathrm{~s})$ ions is smaller than for the $\mathrm{N}(1 \mathrm{~s})$ ions. The energy change due to many-body effects for the CAS(6,7) wavefunctions for the $\mathrm{N}(1 \mathrm{~s})$ ions is $4.8 \mathrm{eV}$ while for the $\mathrm{O}(1 \mathrm{~s})$ ions, it is only $2.5 \mathrm{eV}$ or smaller by almost a factor of 2; see Tables 5 and 6. This is consistent with the HF being a better description when the charge separation is greater, as between $\mathrm{N}$ and the equivalent core $\mathrm{F}^{+}$. Second, the properties of the CAS wavefunctions are more similar between the ${ }^{3} \Pi$ and ${ }^{1} \Pi$ multiplets for the $\mathrm{O}(1 \mathrm{~s})$ ions than for the $\mathrm{N}(1 \mathrm{~s})$ ions; compare the occupations, $<\mathrm{z}>$, and $\mathrm{E}_{\mathrm{corr}}$ in Tables 5 and 6 . Taken together, the importance of many body effects for the relative intensities of the multiplets is not especially important for the $\mathrm{O}(1 \mathrm{~s})$ ions.

\section{Conclusions}

While HF wavefunctions provide a reasonable, qualitative prediction of the XPS features of the open shell NO molecule, it is important to take into account many-body effects to get more quantitative information. The analysis of many-body effects presented in this paper has stressed the physical and chemical content of the important many body effects. Thus, rather than looking to determine the CI wavefunction [41] or the density functional that gives the most accurate BEs, [4,39] modest errors have been accepted provided that the important many-body effects have been explicitly identified. In particular, the analysis has focused on static correlation effects that involve orbitals and configurations that are nearly degenerate. A particular advantage of this approach is that it can be generalized to more complex situations involving the XPS of molecules adsorbed on surfaces and the XPS of condensed matter systems [5].

For $\mathrm{NO}$, we have found that there is a significant differential many-body effect between the different states involved in our study, the ground state, the $\mathrm{N}(1 \mathrm{~s})$ hole, and the $\mathrm{O}(1 \mathrm{~s})$ hole configurations. The many body effects are most important for the $\mathrm{N}(1 \mathrm{~s})$ hole, intermediate for the ground state, and least important for the $\mathrm{O}(1 \mathrm{~s})$ hole. The consequences of these differential effects are that they reduce the N(1s) BEs from their HF values while they increase the O(1s) BEs from their HF values. Furthermore, while the relative XPS intensity of the high and low spin multiplets of the $\mathrm{N}(1 \mathrm{~s})$ ion changes considerably going from HF to CAS wavefunctions, where 
many body effects are included, this relative intensity does not change significantly for the $\mathrm{O}(1 \mathrm{~s})$ ionic multiplets. The differential correlation effects can be rationalized with the use of the equivalent core approximation [17,18] where the core ionized atom is replaced by the next higher element in the periodic table. Direct calculations comparing core-hole wavefunctions with equivalent core approximation wavefunctions have been used to demonstrate the qualitative validity of the model and to justify its use to rationalize the differential correlation effects. The correlation that we are able to make with the concepts of the equivalent core approximation is that the static many body effects are largest when the charge separation between the atoms is smallest and largest when the charge separation is greatest. Thus the $\mathrm{N}(1 \mathrm{~s})$ core hole with the equivalent core model of $\mathrm{O}_{2}^{+}$has zero charge separation while the $\mathrm{O}(1 \mathrm{~s})$ core hole with the equivalent core model of $\mathrm{NF}^{+}$has the largest charge separation of 2 . Thus, the changes in the charge separation are fully consistent with the calculated differential many-body effects. In addition, we have characterized the important many body effects as involving excitations among bonding and anti-bonding orbitals involving the $1 \pi$ and $2 \pi$ and the $5 \sigma$ and $6 \sigma$ pairs of orbitals.

The advantage of studying the XPS of an isolated, and small, molecule is that it is possible to make detailed tests to identify features that may also be applicable for more complex systems. Thus, the importance of making parallel treatments of the static correlation effects for the initial, ground state and the final, core ion configurations has been clearly demonstrated in this study of NO and clearly merits further study for condensed systems, especially oxides, where it has not always been applied; see the review in Ref. [5]. A novel feature of the present work involves the use of CAS wavefunctions to determine pairs of bonding and anti-bonding orbitals which are optimized to represent static many body effects in the initial and final state wavefunctions. The development of theoretical and computational methods to use such optimized orbitals may well be useful for the analysis of the main and satellite features of the XPS of ionic compounds. Furthermore, the correlation between the importance of many-body effects and the charge separation of the constituent atoms has not been applied to interpret the XPS of condensed systems. In order to make this correlation, it will be necessary to generalize the concept of equivalent cores for the analysis of the XPS of metal oxides. This is because the concern is to understand the effective charges seen by the valence electrons when the atoms in a compound have very different nuclear charges as is the case for many metal oxides, and this is not as direct as increasing the nuclear charge of the core ionized atom by one. However, it should be possible to define these effective charges based on the sizes, as given by the expectation values of r, of 
atomic orbitals for the different atoms in a compound with or without core ionization. The correlation of many-body effects and effective nuclear charges has not been examined for the XPS of metal oxides. The present study strongly suggests that such a correlation, both for qualitative analysis and interpretation as well as for direct theoretical computation, is certainly merited.

\section{Acknowledgements.}

PSB acknowledge support from the U.S. Department of Energy, Office of Science, Office of Basic Energy Sciences, Chemical Sciences, Geosciences, and Biosciences (CSGB) Division through the Geosciences program at Pacific Northwest National Laboratory. CS and FI have been supported by the Spanish MINECO/FEDER through CTQ-2015-64618-R and Excellence María de Maeztu program MDM-2017-0767 grants and, in part, by Generalitat de Catalunya grants 2014SGR97 and XRQTC. F.I. acknowledges additional support from the 2015 ICREA Academia Award for Excellence in University Research. 


\section{Table Captions:}

Table 1. Relativistic contributions, $\Delta \mathrm{BE}_{\text {rel }}$ in $\mathrm{eV}$, for the $1 \mathrm{~s} \mathrm{BEs}$ of $\mathrm{NO}$ and the $\mathrm{N}$ and $\mathrm{O}$ atoms; the values are for the average of the open shell configurations; see text.

Table 2. Theoretical, HF, CAS(4,5) and CAS(6,7), N(1s) and $\mathrm{O}(1 \mathrm{~s}) \mathrm{BEs}$, in $\mathrm{eV}$, for the ${ }^{3} \Pi$ and ${ }^{1} \Pi$ multiplets; the multiplet splitting of the BEs, $\Delta\left({ }^{1} \Pi^{-}{ }^{3} \Pi\right)$ are also given. These BEs are compared to experiment.

Table 3. XPS $I_{\text {rel }}$ for the N(1s) ${ }^{3} \Pi$ and ${ }^{1} \Pi$ multiplets obtained with HF and CAS wavefunctions compared to the ideal intensities normalized to the multiplicity of the ions. The losses, in percent, to shake excitations and the ratio of the multiplet intensities are also given.

Table 4. XPS $I_{\text {rel }}$ for the $\mathrm{O}(1 \mathrm{~s}){ }^{3} \Pi$ and ${ }^{1} \Pi$ multiplets obtained with HF and CAS wavefunctions; see caption to Table VII. 
Table 1. Relativistic contributions, $\Delta \mathrm{BE}_{\text {rel }}$ in $\mathrm{eV}$, for the $1 \mathrm{~s} \mathrm{BEs}$ of $\mathrm{NO}$ and the $\mathrm{N}$ and $\mathrm{O}$ atoms; the values are for the average of the open shell configurations; see text.

\begin{tabular}{|l|l|l|}
\hline & \multicolumn{2}{|c|}{$\Delta \mathrm{BE}_{\text {rel }}$} \\
\hline & NO & Atom \\
\hline $\mathrm{N}(1 \mathrm{~s})$ & 0.25 & 0.25 \\
\hline $\mathrm{O}(1 \mathrm{~s})$ & 0.47 & 0.45 \\
\hline
\end{tabular}


Table 2. Theoretical, HF, CAS(4,5) and CAS(6,7), N(1s) and $\mathrm{O}(1 \mathrm{~s}) \mathrm{BEs}$, in $\mathrm{eV}$, for the ${ }^{3} \Pi$ and ${ }^{1} \Pi$ multiplets; the multiplet splitting of the BEs, $\Delta\left({ }^{1} \Pi^{-3} \Pi\right)$ are also given. These BEs are compared to experiment.

\begin{tabular}{|l|c|c|l|l|}
\hline & HF & CAS(4,5) & CAS(6,7) & Experiment $^{\text {a }}$ \\
\hline & \multicolumn{5}{|c|}{ BE[N(1s)] } \\
\hline${ }^{3} \Pi$ & 411.53 & 410.26 & 409.71 & 410.3 \\
\hline${ }^{1} \Pi$ & 412.89 & 411.51 & 411.01 & 411.8 \\
\hline$\Delta\left({ }^{1} \Pi-{ }^{3} \Pi\right)$ & 1.36 & 1.27 & 1.30 & $1.412 \pm 0.016$ \\
\hline & & & & \\
\hline${ }^{3} \Pi$ & 542.61 & 543.37 & 543.06 & 543.3 \\
\hline${ }^{1} \Pi$ & 543.10 & 543.85 & 543.51 & 544.0 \\
\hline$\Delta\left({ }^{1} \Pi-{ }^{3} \Pi\right)$ & 0.49 & $\ldots 0.48$ & $\ldots 0.46$ & $\ldots 0.530 \pm 0.021$ \\
\hline
\end{tabular}

${ }^{a}$ Experimental data taken from Ref. [31] 
Table 3. XPS $I_{\text {rel }}$ for the N(1s) ${ }^{3} \Pi$ and ${ }^{1} \Pi$ multiplets obtained with HF and CAS wavefunctions compared to the ideal intensities normalized to the multiplicity of the ions. The losses, in percent, to shake excitations and the ratio of the multiplet intensities are also given.

\begin{tabular}{|l|l|l|l|}
\hline & \multicolumn{3}{|c|}{ SA Intensity } \\
\hline & $\mathrm{I}_{\mathrm{rel}}\left[{ }^{3} \Pi\right]-(\mathrm{loss})$ & $\mathrm{I}_{\mathrm{rel}}\left[{ }^{1} \Pi\right]-(\mathrm{loss})$ & Ratio \\
\hline Ideal & 3.00 & 1.00 & 3.00 \\
\hline HF & $2.28(24 \%)$ & $0,74(26 \%)$ & 3.09 \\
\hline CAS(4,5) & $2.19(27 \%)$ & $0.64(36 \%)$ & 3.41 \\
\hline CAS(6,7) & $2.15(29 \%)$ & $0.65(35 \%)$ & 3.30 \\
\hline Experiment ${ }^{\mathrm{a}}$ & ---- & ---- & $3.43 \pm 0.08$ \\
\hline
\end{tabular}

${ }^{\mathrm{a}}$ Ref. [16]

Table 4. XPS $I_{\text {rel }}$ for the $\mathrm{O}(1 \mathrm{~s}){ }^{3} \Pi$ and ${ }^{1} \Pi$ multiplets obtained with HF and CAS wavefunctions; see caption to Table 3.

\begin{tabular}{|l|l|l|l|}
\hline & \multicolumn{3}{|c|}{ SA Intensity } \\
\hline & $\left.I_{\text {rel }}{ }^{3} \Pi\right]-($ loss $)$ & $\left.I_{\text {rel[ }}^{1} \Pi\right]-(l o s s)$ & Ratio \\
\hline Ideal & 3.00 & 1.00 & 3.00 \\
\hline HF & $2.18(27 \%)$ & $0,71(29 \%)$ & 3.08 \\
\hline CAS(4,5) & $2.11(30 \%)$ & $0.68(32 \%)$ & 3.11 \\
\hline CAS(6,7) & $2.06(31 \%)$ & $0.66(34 \%)$ & 3.12 \\
\hline
\end{tabular}




\section{References}

1 Siegbahn K, Nordling C, Johansson G, Hedman J, Hedén P F, Hamrin K, Gelius U, Bergmark T, Werme L O, Manne R, Baer Y (1969) ESCA-Applied to Free Molecules (North-Holland, Amsterdam)

2 Siegbahn K, Nordling C, Fahlman A, Nordberg R, Hamrin K, Hedman J, Johansson G, Bergmark T, Karlsson S E, Lindgren I, Lindberg B (1967) ESCA-Atomic, Molecular, and Solid State Structure Studied by Means of Electron Spectroscopy (Almqvist and Wiksells, Uppsala, Sweden).

3 Bagus PS (1965) Self-Consistent-Field Wave Functions for Hole States of Some Ne-Like and Ar-Like Ions. Phys Rev 139:A619.

4 Pueyo Bellafont N, Bagus P S, Illas F (2015) Prediction of core level binding energies in density functional theory: Rigorous definition of initial and final state contributions and implications on the physical meaning of Kohn-Sham energies. J Chem Phys 142:214102.

5 Bagus P S, Ilton E S, Nelin CJ (2013) The Interpretation of XPS Spectra: Insights Into Materials Properties. Surf Sci Rep 68:273.

6 Bagus P S, Ilton E S, Nelin C J (2018) Extracting Chemical Information From XPS Spectra: A Perspective. Catal Lett 148:1785.

${ }^{7}$ R. P. Gupta and S. K. Sen, "Calculation of multiplet structure of core p-vacancy levels. II", Phys. Rev. B 12, 15 (1975).

8 Gupta R P, Sen S K (1974) Calculation of multiplet structure of core p-vacancy levels. Phys Rev B 10:71.

9 Bagus P S, Freeman A J, Sasaki F (1973) Prediction of new multiplet structure in photoemission experiments. Phys Rev Lett 30:850.

10 Bagus P S, Broer R, Ilton E S (2004) A new near degeneracy effect for photoemission in transition metals. Chem Phys Lett 394:150.

11 Valderrama E, Ludena E V, Hinze J (1999) Assessment of dynamical and nondynamical correlation energy components for the beryllium-atom isoelectronic sequence, J Chem Phys 110: 2343 .

12 Knowles P, Schütz M, Werner H -J (2000) Ab Initio Methods for Electron Correlation in Molecules in Modern Methods and Algorthms of Quantum Chemistry, edited by J. Grotendorst (John von Neumann Institute for Computing, Jülich) Vol. 3 p. 97. 
13 Bagus P S, Broer R, Parmigiani F (2006) Anomalous electron correlation due to near degeneracy effects: low-lying ionic states of Ne and Ar. Chem Phys Lett 421:148.

14 Bagus P S, Sousa C, Illas F (2016) Consequences of electron correlation for XPS binding energies: Representative case for C(1s) and O(1s) XPS of CO. J Chem Phys 145:144303.

15 Mulliken R S (1949) J Chim Phys 46:497.

16 Bagus P S, Schrenk M, Davis D W, Shirley D A (1974) Anomalous multiplet-splitting intensity ratios in K-level X-ray photoemission. Phys Rev A9:1090.

17 Koepke J W, Jolly W L (1976) The core electron binding energies of oxygen difluoride. J Electron Spectrosc Relat Phenom 9:413.

18 Jolly W L (1972) The estimation of core-electron binding-energy shifts using the concept of the equivalence of equally charged cores, in Proceedings of the international conference on electron spectroscopy, edited by D. A. Shirley (North-Holland, Amsterdam, Netherlands), p. 629.

19 Viñes F, Sousa C, Illas F (2018) On the prediction of core level binding energies in molecules, surfaces and solids. Phys Chem Chem Phys 20:8403.

20 Levine IN (2000) Quantum Chemistry (Prentice-Hall, Upper Saddle River, NJ)

21 van Setten M J, Costa R, Viñes F, Illas F (2018) Assessing GW approaches for predicting core level binding energies.J Chem Theory Comput 14:877.

22 Deleuze MS, Cederbaum LS (1999) The new challenges of the theory of ionization for polymers and solids. Adv. Quantum Chem 35:77

23 Ortiz JV (2013) Electron propagator theory: an approach to prediction and interpretation in quantum chemistry. Wiley Interdisciplinary Reviews-Computational Molecular Science. 2:123

24 Roos B O, Taylor P R, Siegbahn P E M (1980) A complete active space SCF method (CASSCF) using a density matrix formulated super-CI approach. Chem Phys 48:157.

25 Manne R, Åberg T (1970) Koopmans' theorem for inner-shell ionization. Chem Phys Lett $7: 282$.

26 Aberg T (1967) Theory of X-Ray Satellites. Phys Rev 156:35.

27 Pueyo Bellafont N, Viñes F, Illas F (2016) Performance of the TPSS Functional on Predicting Core Level Binding Energies of Main Group Elements Containing Molecules: A Good Choice for Molecules Adsorbed on Metal Surfaces, J Chem Theory Comput 12:324. 
28 Visscher L, Visser O, Aerts P J C, Merenga H, Nieuwpoort WC (1994) Relativistic quantum chemistry: The MOLFDIR program package. Comput Phys Commun 81:120.

29 DIRAC, a relativistic ab initio electronic structure program, Release DIRAC08 (2008), written by Visscher L, Jensen H J Aa, Saue T, with new contributions from Bast R, Dubillard S, Dyall K G, Ekström U, Eliav E, Fleig T, Gomes A S P, Helgaker T U, Henriksson J, Iliaš M, Jacob Ch R, Knecht S, Norman P, Olsen J, Pernpointner M, Ruud K, Sałek P, Sikkema J (see the URL at http://dirac.chem.sdu.dk).

30 Herzberg G (1950) Molecular Spectra and Molecular Structure Vol. I (Van Nostrand, Princeton)

31 Bagus P S, Schaefer HF (1971) Direct Near-Hartree--Fock Calculations on the 1s Hole States of $\mathrm{NO}^{+}$. J Chem Phys 55:1474.

32 Clementi E, Roothaan C C J, Yoshimine M (1962)Accurate Analytical Self-Consistent Field Functions for Atoms. II. Lowest Configurations of the Neutral First Row Atoms. Phys Rev 127:1618.

33 Duijneveldt FB (1971) IBM Internal Report RJ945.

34 Partridge H (1989) Near Hartree-Fock quality GTO basis sets for the first- and third-row atoms. J Chem Phys 90:1043.

35 Huber K P, Herzberg G (1979) Molecular Spectra and Molecular Structure Vol. 4, Constants of Diatomic Molecules (Van Nostrand, New York).

36 Löwdin P O (1975) Quantum Theory of Many-Particle Systems. I. Physical Interpertations by Means of Density Matrices, Natural Spin-Orbitals, and Convergence Problems in the Method of Configurational Interaction. Phys Rev 97:1474.

37 Prosser F, Hagstrom S (1968) Corresppondin g Orbitals and Cofactors. J Chem Phys 48:4807. 38 CLIPS is a program system to compute ab initio SCF and correlated wavefunctions for polyatomic systems. It has been developed based on the publicly available programs in the ALCHEMY package from the IBM San Jose Research Laboratory by Bagus P S, Liu B, McLean A D and Yoshimine M.

39 Pueyo Bellafont N, Illas F, Bagus P S (2015) Validation of Koopmans' Theorem For Density Functional Theory Binding Energies. Phys Chem Chem Phys 17:4015.

40 Bagus P S, Ilton E S (2006) Effects of covalency on the p-shell photoemission of transition metals: MnO. Phys Rev B73:155110. 
41 de Graaf C, Sousa C, Broer R (1999) Ionization and excitation energies in $\mathrm{CuCl}$ and $\mathrm{NiO}$ within different embedding schemes. J. Mol Struc THEOCHEM 458:53. 